C. Pérez-González, M. Colebrook, J.L. Roda-García, P. González Yanes, T. González Dos Santos, V. Plaza Martín TARO-ULL Research Group,

Universidad de La Laguna

C. Rosa Remedios

Centro Coordinador de Emergencias

y Seguridad 1-1-2 (CECOES)

\title{
Analysis of incidents registered by the 1-1-2 Canarias emergency services by using data science techniques with the $R$ software
}

The study of alerts received in the emergency services is a very important issue in order to know different aspects of the time and spatial distribution of alerts in a given region. In particular, the Emergency and Security Coordinating Center (CECOES) 1-1-2 of the Government of the Canary Islands records detailed information about the incidents that are reported by the citizens through phone calls. Due to the high volume of information generated over the time in this process, it is needed to apply big data techniques to obtain statistical measures and results of interest. We have used the statistical software R and different libraries (Shiny, Highcharts, Highmaps) to present the data information in different interactive dashboards (time series charts to analyze the time evolution, geospatial representations of incidents density distribution, etc.). In this work we illustrate some of these charts that help the public authorities to study the incidents in the region during the last years. 


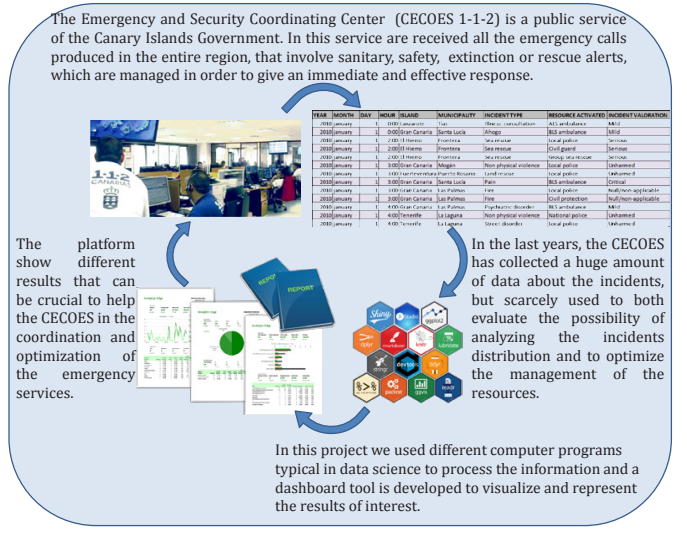

This dashboard shows again multiple options to compare, in this case, the average number
of incidents between different years, months or, even, the time in the day.

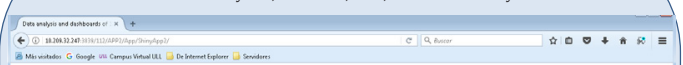
Data analysis and dashboards of 1-1-2 Canarias

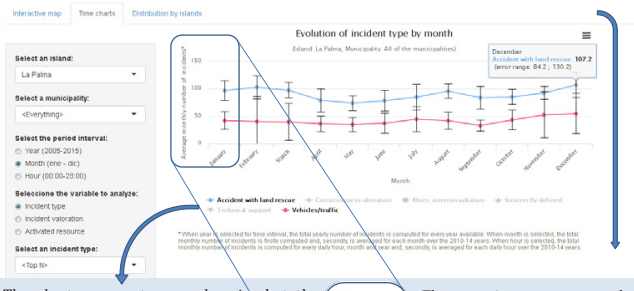

The chart represents several series but the The margin errors around the user can select (over the chart legend) only _- mean (represented as intervals) those to be shown. The time series charts allow us to detect - about the accuracy in computing changes or identify patterns, that is very to the averages.

important in predicting potential future - If the values are over dispersed, the demands of the resources or, even, changes in $(2$, mater average is imprecise.

municipalities.

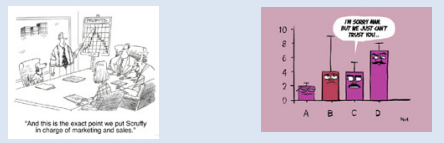

Let's suppose that we are interested to compare the monthly average number of incidents that were associated to critical or exitus assesment in Arrecife (Lanzarote)

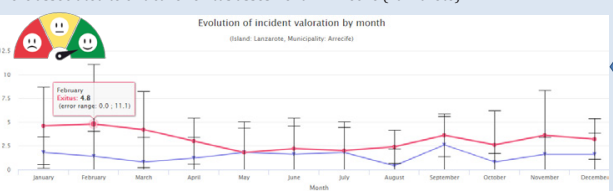

In this case, the example shows the average number of incidents along the day where the activated resources were ALS ambulances (advanced life support) and the local police in Las Palmas (Gran Canaria)
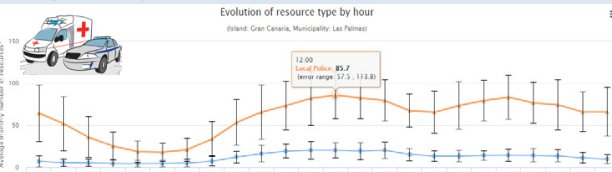

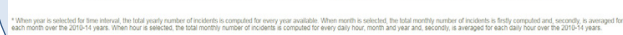

Title:

Analysis of incidents registered by the 1-1-2 Canarias emergency services Authors:

Colebrook Santamaría, M. ${ }^{1}$; González Dos Santos, T. ${ }^{1}$; González Yanes, P. ${ }^{1}$, Pérez-González, C. J. ${ }^{1}$; Plaza Martín, V. ${ }^{1}$;Roda García, J. L. ${ }^{1}$; Rosa Remedios, C. ${ }^{2}$; Affiliation:

(1) Grupo de investigación TARO-ULL (Universidad de La Laguna) (email: cpgonzal@ull.es) ,

(2) Centro Coordinador de Emergencias y Seguridad 1-1-2 (CECOES).

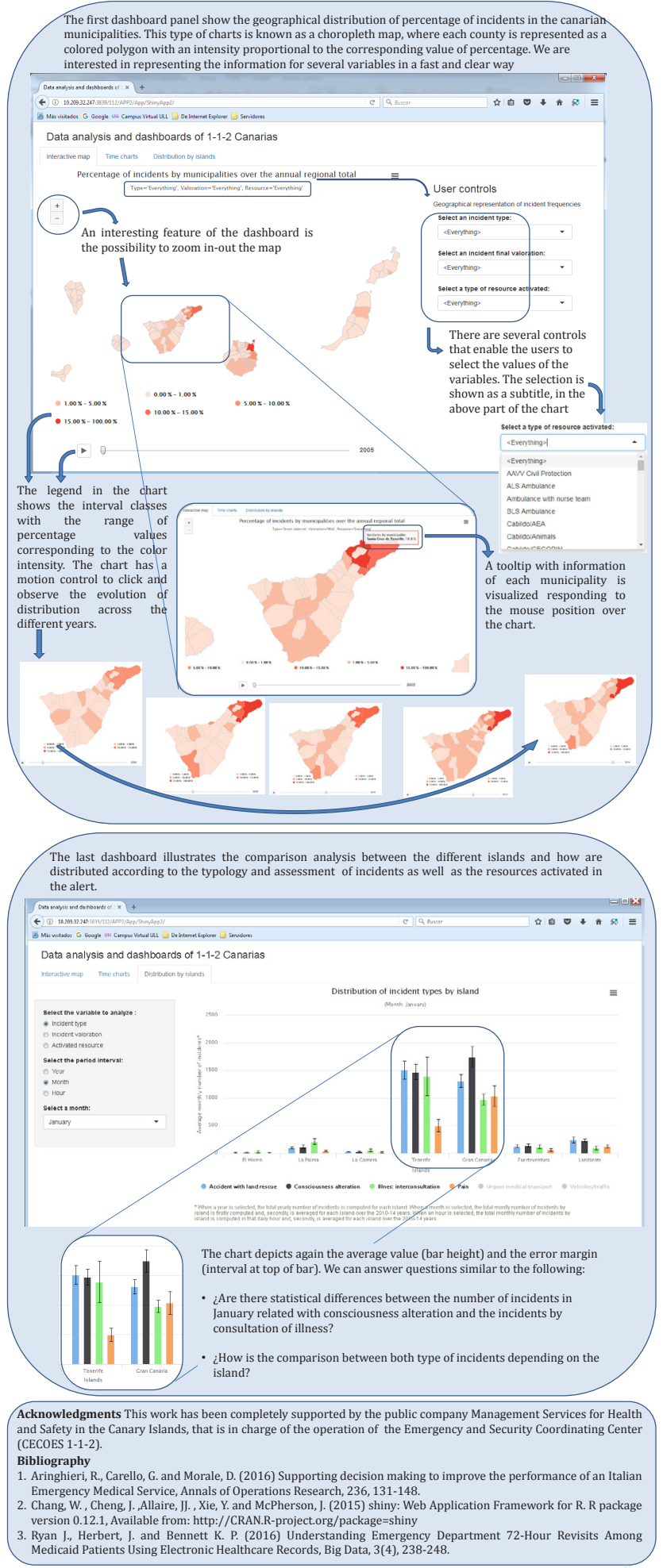

Medicaid Patients Using Electronic Healthcare Records, Big Data, 3(4), 238-248. 